INPLASY

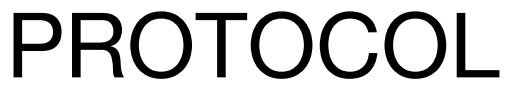

To cite: Chu et al. The efficacy and safety of fire needle therapy for alopecia areata: a protocol for systematic review and meta-analysis. Inplasy protocol 202220053. doi: 10.37766/inplasy2022.2.0053

Received: 14 February 2022

Published: 14 February 2022

Corresponding author: Yixin Zhang

hbzyx@163.com

Author Affiliation:

This systematic review belongs to School of Pharmacy, Hebei University of Chinese Medicine and Guang'anmen Hospital, China Academy of Chinese Medical Science.sGuang'anmen Hospital, China Academy of Chinese Medical Science.

Support: Cl2021A02306.

Review Stage at time of this submission: The review has not yet started.

Conflicts of interest:

None declared.

\section{The efficacy and safety of fire needle therapy for alopecia areata: a protocol for systematic review and meta-analysis}

Chu, XQ1; Biao, YN2; Guo, YF3; Liu, CX4; Zhao, HL5; Zhang, YX66.

Review question / Objective: To assess the benificial effect and safety of fire needle therapy for alopecia areata.

Condition being studied: Alopecia areata (AA) is a common autoimmune disease, targeting hair follicles in the anagen phase. It results in non-scarring alopecia, and those affected respond primarily with camouflage; topical, intralesional, or systemic corticosteroids; minoxidil; diphenylcyclopropenone; or systematic agents. Randomized controlled trials (RCTs) to date suggest that fire needle therapy could potentially be used to treat AA; however, evidence-based medical confirmation is lacking. We therefore systematically evaluated the efficacy and safety of fire needle therapy for the treatment of AA.

Information sources: PubMed, Web of Science, Cochrane, Embase, Sinomed, China National Knowledge Infrastructure, Chongqing VIP Information, and WanFang Data. ClinicalTrials.gov and Chinese Clinic Trials.gov .

INPLASY registration number: This protocol was registered with the International Platform of Registered Systematic Review and Meta-Analysis Protocols (INPLASY) on 14 February 2022 and was last updated on 14 February 2022 (registration number INPLASY202220053).

\section{INTRODUCTION}

Review question / Objective: To assess the benificial effect and safety of fire needle therapy for alopecia areata.
Condition being studied: Alopecia areata (AA) is a common autoimmune disease, targeting hair follicles in the anagen phase. It results in non-scarring alopecia, and those affected respond primarily with camouflage; topical, intralesional, or 
systemic corticosteroids; minoxidil; diphenylcyclopropenone; or systematic agents. Randomized controlled trials (RCTs) to date suggest that fire needle therapy could potentially be used to treat AA; however, evidence-based medical confirmation is lacking. We therefore systematically evaluated the efficacy and safety of fire needle therapy for the treatment of AA.

\section{METHODS}

Participant or population: Patients were diagnoesd with alopecia areata.

Intervention: Those where in the experiment group was subjected to fire needle therapy, whereas the controls were treated using other therapeutic methods. Those where in the the experiment group was subjected to fire needle therapy in combination with alternative treatment, whereas the controls were treated only using the identical alternative treatment.The treatment group was fire needle therapy.

Comparator: The conparison group consisted of those receiving routine care or any intervention other than fire needle therapy.

Study designs to be included: Our team utilized all RCTs assessing the potency and safeness of fire needle therapy for AA no matter the language, published or unpublished.

Eligibility criteria: We will include all RCTs evaluating the efficacy and safety of fire needle therapy for AA no matter the language, whether published or not. Randomized crossover trials, animal trials, medical cases, and studies published only as abstracts will be excluded.

Information sources: PubMed, Web of Science, Cochrane, Embase, Sinomed, China National Knowledge Infrastructure, Chongqing VIP Information, and WanFang Data. ClinicalTrials.gov and Chinese Clinic Trials.gov .
Main outcome(s): The average difference in score on the Severity of Alopecia Tool (SALT) after treatment, compared to before treatment; Dermoscopic assessment; Satisfaction with the appearance of hair; Incidence of adverseevents.

Quality assessment / Risk of bias analysis: Two reviewers (XQC/YNB) will separately assess risk-of-bias using the guidelines in Cochrane Reviewer's Handbook (v. 5.0.24). This tool in included studies will be evaluated according the following domains: blinding of participants, allocation concealment, random sequence generation, blinding of participants and outcome assessors selective reporting, incomplete outcome data, and other bias. Each will be classified as "low," "unclear," or "high" based on information provided by the trials. The evaluated domains will be evaluated separately by 2 reviews, and differences will be resolved by a third reviewer.

Strategy of data synthesis: Heterogeneity will be assessed using the guidelines in the RevMan software. The value of 12 will be used for heterogeneity calculations. Either the random-effect or mixed-effect model will be adopted (when 12 ( $50 \%$ [signin e adopteddeletero] or < 50\% [minor heterogeneity], respectively). When either substantial or considerable heterogeneity is detected, subgroup analysis and sensitivity analysis will be performed to determine possible causes.

Subgroup analysis: If the necessary data are available, subgroup analysis will be performed, focusing on type of $A A$, treatment period, type of fire needle therapy, and type of intervention in the control or study group.

Sensitivity analysis: In the case of important heterogeneity, a sensitivity analysis will be performed, focusing on heterogeneity qualities, sample size, study characteristics, and methodological element. If heterogeneity is reduced by excluding small and low-quality studies, our conclusion will be made with care. 
Language: No language limits.

Country(ies) involved: China.

Keywords: Alopecia areata, fire needle, meta-analysis, protocol, systematic review.

Contributions of each author:

Author 1 - Xin qiao Chu - The author drafted the manuscript.

Author 2 - Yaning Biao - The author provided statistical expertise.

Author 3 - Yufeng Guo - The author contributed to the development of the selection criteria, and the risk of bias assessment strategy.

Author 4 - Chenxu Liu - The author read, provided feedback and approved the final manuscript.

Author 5 - Hanlin Zhao - The author contributed to the development of the selection criteria, and the risk of bias assessment strategy.

Author 6 - Yixin Zhang - The author provided statistical expertise. 